\title{
Article \\ Estimation of Plant Height and Aboveground Biomass of Toona sinensis under Drought Stress Using RGB-D Imaging
}

\author{
Wenjian Liu, Yanjie Li $* \mathbb{D}$, Jun Liu and Jingmin Jiang \\ Research Institute of Subtropical Forestry, Chinese Academy of Forestry, Hangzhou 311400, China; \\ 17854262061@163.com (W.L.); ucjackley@gmail.com (J.L.); jiangjm23@gmail.com (J.J.) \\ * Correspondence: aj7105@gmail.com
}

Citation: Liu, W.; Li, Y.; Liu, J.; Jiang, J. Estimation of Plant Height and Aboveground Biomass of Toona sinensis under Drought Stress Using RGB-D Imaging. Forests 2021, 12, 1747. https://doi.org/10.3390/f12121747

Academic Editor: Jarosław Socha

Received: 16 November 2021

Accepted: 9 December 2021

Published: 10 December 2021

Publisher's Note: MDPI stays neutral with regard to jurisdictional claims in published maps and institutional affiliations.

Copyright: (C) 2021 by the authors. Licensee MDPI, Basel, Switzerland. This article is an open access article distributed under the terms and conditions of the Creative Commons Attribution (CC BY) license (https:// creativecommons.org/licenses/by/ $4.0 /)$.

\begin{abstract}
Rapid and accurate plant growth and biomass estimation is essential for formulating and implementing targeted forest cultivation measures. In this study, RGB-D imaging technology was used to obtain the RGB and depth imaging data for a Toona sinensis seedling canopy to estimate plant growth and aboveground biomass (AGB). Three hundred T. sinensis seedlings from 20 varieties were planted under five different drought stress treatments. The U-Net model was applied first to achieve highly accurate segmentation of plants from complex backgrounds. Simple linear regression (SLR) was used for plant height prediction, and the other three models, including multivariate linear (ML), random forest (RF) and multilayer perceptron (MLP) regression, were applied to predict the AGB and compared for optimal model selection. The results showed that the SLR model yields promising and reliable results for the prediction of plant height, with $\mathrm{R}^{2}$ and RMSE values of 0.72 and $1.89 \mathrm{~cm}$, respectively. All three regression methods perform well in the prediction of AGB estimation. MLP yields the highest accuracy in predicting dry and fresh aboveground biomass compared to the other two regression models, with $\mathrm{R}^{2}$ values of 0.77 and 0.83 , respectively. The combination of Gray, Green minus red (GMR) and Excess green index (ExG) was identified as the key predictor by RReliefF for predicting dry AGB. GMR was the most important in predicting fresh AGB. This study demonstrated that the merits of RGB-D and machine learning models are effective phenotyping techniques for plant height and AGB prediction, and can be used to assist dynamic responses to drought stress for breeding selection.
\end{abstract}

Keywords: RGB-D imaging; Toona sinensis seedling; aboveground biomass; plant height; machine learning

\section{Introduction}

Toona sinensis, which belongs to the Meliaceae family, is a fast-growing deciduous tree with a straight trunk, hard wood and beautiful texture [1]. In addition, T. sinensis is also known as a nutritious healthy vegetable with young buds and leaves rich in protein, fat, minerals and vitamins [2]. Furthermore, T. sinensis has also been widely used in traditional Chinese medicine as the leaves are rich in limonoids, flavonoids and terpenoid components that have antibacterial, hypoglycemic, lipid-lowering and anti-cancer effects [3,4]. T. sinensis also has the characteristics of drought resistance and endure wetness, which can be used for ecological improvement [2]. Studies have shown that the vigorous growth period of $T$. sinensis is from June to September each year. This season is prone to continuous drought [5]. In order to effectively prevent the seasonal and continuous drought from harming T. sinensis seedlings and meet the growing market demand, it is of great significance to monitor the growth and nutritional status of $T$. sinensis seedlings dynamically in real time to ensure that high-quality $T$. sinensis varieties are selected.

Plant height $(\mathrm{H})$ and AGB are two important parameters that characterize the growth of T. sinensis. AGB is not only closely related to the nutritional status of forest trees, plant survival adaptability and production capacity but also an important parameter for 
calculating biological carbon storage [6,7]. In different habitats, plants can adapt to the external environment by adjusting the distribution pattern of biomass in various organs to make better use of limited resources to maintain their own survival and growth [8]. Therefore, the real-time dynamic monitoring of the growth and nutritional status of $T$. sinensis without destructive collection is of great significance to ensure that high-quality T. sinensis is selected as a vegetable for consumption and for the quantitative analysis of genotype-environment interaction effects, which will help T. sinensis breeding [9].

However, the traditional methods of measuring plant height and aboveground biomass of seedlings require destructive sampling and manual measurement, which are extremely time-consuming $[10,11]$. In addition, these measurement methods are greatly affected by the subjective factors of the experimenter and have problems such as low efficiency and heavy workload, which cannot meet the requirements of high throughput, automated plant phenotype analysis needs, and inability to carry out real-time monitoring [12,13]. Currently, the developed high-throughput phenotyping (HTP) innovations, including unmanned aerial vehicles (UAVs), small high-resolution camera systems (standard RGB, multispectral or hyperspectral) and image-derived indices, are shown to be promising tools for carrying out real-time monitoring of plants $[7,14,15]$. In particular, remote sensing has become the main data source for large-area AGB estimation $[7,16]$. However, remote sensing and UAV spectral data collection require the use of special equipment and global positioning system (GPS) parameters [17], which is not suitable for the estimation of plant traits on small-scale potted greenhouses. RGB-depth (RGB-D) cameras can not only capture color images of objects but also provide depth image information [18] and hold the advantages of low cost, high resolution and convenient data acquisition $[19,20]$, which has been widely used by many researchers to apply RGB-D 3D spatial information for plant phenotype detection [18,21,22]. For instance, Yu et al. [18] obtained color and depth images of litchi fruits through an RGB-D camera and built a random forest model for the classification of litchi fruits. To obtain the RGB-D data of a single plant, Yang et al. [14] used the VCCS + LCCP method to divide the seedlings and used three-dimensional sliding windows and the SVM algorithm to predict the growth parameters of greenhouse crops at the seedling stage. Furthermore, the visible spectrum index (CIVE, ExG, Excess green minus excess red (ExGR), Normalized green-red difference index (NGRDI), etc.) has been used to construct partial least square regression (PLSR), BP neural network (BPNN), support vector machine regression (SVR) and other predictive models to evaluate corn aerial parts biomass and yield a reliable accuracy with $R^{2}$ value of $0.82[23,24]$.

Traditional plant phenotypic parameter estimation is based on a linear/nonlinear statistical regression model established between vegetation-related parameters and actual measured data [25,26]. In this process, the problem of multicollinearity will affect the accuracy of the model [24]. In recent years, researchers have found that machine learning methods are more suitable for estimating vegetation parameters, especially in the application of multisource fusion data [24,26]. For example, Nguyen and Lee [27] used hyperspectral canopy reflectance and partial least square regression (PLSR) to assess rice leaf growth and nitrogen status, and the random forest (RF) regression algorithm has been used to estimate the high-density biomass of wetland vegetation [28]. Most studies have also determined the best prediction model performances by comparing multivariate linear (ML) regression, support vector regression (SVR), multilayer perceptron (MLP) and other methods $[23,25,27]$.

Extracting the target plant from the complex background is a key link in RGB image processing. The essence of target image segmentation is to classify pixels using machine vision and image processing methods to segment plant leaves from a complex background. Current image segmentation technology mostly uses the histogram threshold method and the direct extraction of spectrum and texture information. However, few studies have paid attention to the accurate segmentation of plants using the appropriate deep-learning method $[20,21]$. 
The U-Net model is a U-shaped fully convolutional neural network (CNN) improved on the basis of FCN [29]. It is an end-to-end trained supervised learning network [30] and has been widely used in segmentation tasks such as biomedicine and high-resolution remote sensing images [31]. The greatest advantage of this method is that it retains the original features of the training set in the deep learning method, such as the edge features, texture features and shape features of the segmented objects, and improves the model accuracy $[29,30]$.

Therefore, this study makes full use of the features of easy acquisition and high accuracy of RGB-D imaging, combined with U-Net network image segmentation technology and machine learning methods, to carry out research on the estimation of phenotypic parameters including the plant height and aboveground biomass of $T$. sinensis under drought stress. The detailed objectives of the current research were (1) to estimate the ability of the U-Net model to identify and segment $T$. sinensis seedlings from a complex background and extract the color parameters and depth information of T. sinensis aboveground; (2) to build a prediction model for T. sinensis plant height using depth values from RGB-D data; and (3) to establish a low-cost and high-convenience estimation method for the AGB of T. sinensis seedlings by comparing ML, RF and MLP methods.

\section{Materials and Methods}

\subsection{Study Area and Experimental Design}

The experiment was carried out in a greenhouse at the Research Institute of Subtropical Forestry, Chinese Academy of Forestry in Fuyang, Zhejiang (E 119.57', N 30.03') from May 2021 to August 2021. A total of 240 T. sinensis seeds of 20 varieties from different regions of China were grown in experimental pots $(11 \mathrm{~cm}(\mathrm{~h}) \times$ base $14 \mathrm{~cm}(\mathrm{~d}))$, and each plant was relatively consistent in size. The substrate was a mixture of coconut bran, vermiculite and perlite at a ratio of 3:1:1. In the initial stage, an appropriate amount of organic-inorganic compound fertilizers and water were applied uniformly. After two weeks of growth, the water control treatment started. Four water gradients were generated: (1) watering every day, during which the soil water content in the potted plant was saturated; (2) watering every seven days; (3) watering every 14 days; and (4) watering 28 days. There were three replicate plants for each variety used in each treatment group; in total, 240 plants were used. The reason for these treatments was to ensure that there were significant differences in the growth status of $T$. sinensis seedlings, which was conducive to the accuracy of model construction. The plant height and crown width were measured every week. After two months of drought stress, image information was collected, and destructive sampling was performed.

\subsection{RGB-D Imaging System and Data Acquisition}

Similar to Briglia et al. [17], the schematic of the RGB-D imaging system designed in this study is shown in Figure 1. The imaging chamber was equipped with an Intel RealSense LiDAR camera (L515), computer and LED fill light. The potted plants were placed on a conveyor belt and automatically transported to the imaging chambers directly under the RGB-D camera for image acquisition. The distance between the camera and conveyor belt was approximately $1 \mathrm{~m}$. A visual information collection platform for $T$. sinensis seedlings was developed using Kinect SDK 2.0 + Microsoft Visual Studio (VSCode) under the Windows 10 operating system. This platform could collect color images and depth images in real time. The output resolution of the depth stream was $1024 \times 768$, and the RGB frame resolution was $1920 \times 1080$. 

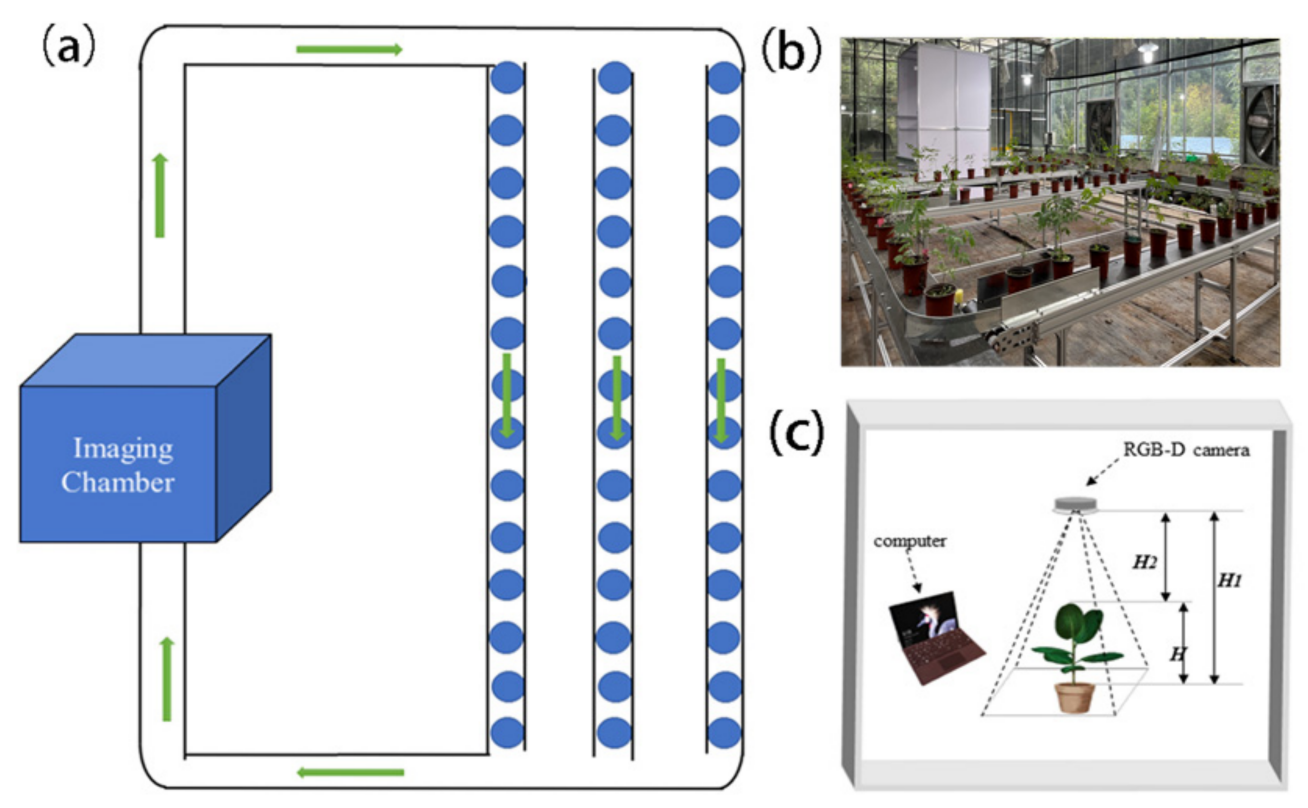

Figure 1. Schematic diagram of the plant phenotyping platform (a), including the location of the RGB-D imaging chamber and the position and moving direction of the flowerpot on the conveyor belt. Front view of the imaging chamber (b) and schematic diagram (c).

Plant height measurements were conducted on the same days as the RGB-D imaging surveys (August 2021) to provide plant truth data [32]. AGB data collection used destructive sampling methods. All of the T. sinensis seedlings on the ground were sampled and weighed for fresh weight (fresh AGB), and then samples were put into envelopes and dried in an oven at $70{ }^{\circ} \mathrm{C}$ to a constant weight. Then, the dry weight (dry AGB) was measured [7].

\subsection{Image Processing and Data Extraction}

\subsubsection{Using U-Net Model of Image Segmentation}

The U-Net network design forms a symmetrical structure by using multiple shrinkage (Left) and expansion (Right) of the image to perform feature learning and establish a feature training library $[30,31]$. The architecture of U-Net is shown in Figure 2. First, 'LabelMe' software was used to mark the area where the T. sinensis seedlings were located, named 'leaf,' as the training sample, for a total of 198. The remaining pictures were used as the validation sample. We input an RGB image, set the width and height to $512 \times 512$, and then set the number of channels to three (R, G, B). The output was set to $Y$, the boundary was filled with 'padding,' and the channel was 1 , which means that the area where the 'leaf' was located was displayed. We optimized the loss function of the model by increasing the weight of the 'leaf'. The output image after convolution has the same resolution as the original image [33]. 


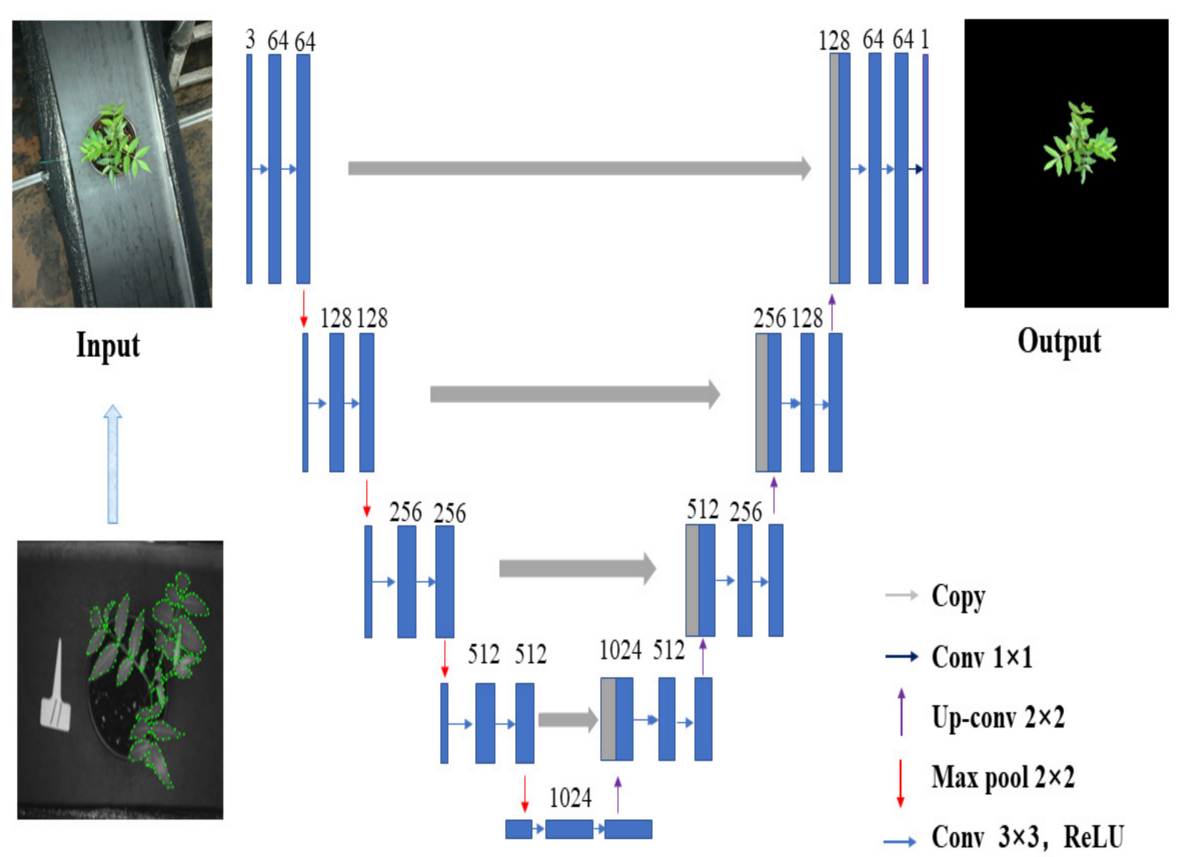

Figure 2. The architecture of U-Net for T. sinensis leaf segmentation.

\subsubsection{Data Extraction}

The red (R), green $(G)$ and blue (B) normalized values of each plant were extracted from the RGB images after segmentation. Subsequently, coordinate system conversion was used to register the depth coordinate space with the color coordinate space to extract the depth value [21]. We used the 'Open3D' visualization software in Python to display the effect of the depth image point cloud. The visible spectrum index of each T. sinensis seedling was calculated using the method found by Li et al. [7] (Table 1). As shown in Figure 1c, the full length of the plant height was calculated as the distance from the base of the plant (the junction of the rhizome) to the tip of the uppermost leaf (Figure 1c). The height was calculated using the following equation:

$$
\text { Height }=\text { Height }_{1}-\text { Height }_{2}
$$

where Height $t_{1}$ is the minimum (distance) depth value from the highest point of the $T$. sinensis seedling to the camera; Height ${ }_{2}$ is the height from the camera to the mouth of the flowerpot; and Height is the plant height.

Table 1. Visible spectrum index calculations.

\begin{tabular}{ccc}
\hline Visible Spectrum Index & Equation & Reference \\
\hline Gray grayscale value (Gray) & $0.3 R+0.59 G+0.11 B$ & {$[20]$} \\
Excess green index (ExG) & $2 G-R-B$ & {$[34]$} \\
Excess red index (ExR) & $1.3 R-G$ & {$[35]$} \\
Excess green minus excess red (ExGR) & $E x G-E x R$ & {$[36]$} \\
Normalized green-red difference & $\frac{G-B}{G+B}$ & {$[37]$} \\
index (NGRDI) & $G-R$ & {$[38]$} \\
Green minus red (GMR) & $\frac{G}{R}$ & {$[39]$} \\
Green-red ratio index (GRRI) & $(R-G)$ & {$[40]$} \\
Normalized difference index (NDI) & {$\left[R+G+0.1^{\times}(R+G+B)\right]$} & \\
\hline
\end{tabular}




\subsection{Data Analysis}

\subsubsection{Linear Regression}

Simple linear regression (SLR) was used to build a predictive model of plant height using the image depth value [15]. Multivariate linear (ML) regression was implemented to build prediction models for fresh AGB and dry AGB [41]. In this study, 360 sets of data were collected for model construction, including $80 \%$ for calibration and $20 \%$ for validation. The stochastic gradient descent (SGD) algorithm was used to update the parameters [42], 500 times were trained to calculate the loss value, and the model slope and intercept and the fitted model were generated [23]. The model loss value was expressed by log mean square error (MSE) loss, also known as L2 loss, and the formula is as follows:

$$
\text { L2 Loss }=\sqrt{\frac{1}{n} \sum_{i=1}^{n}\left(\log \left(y_{i}\right)-\log \left(y_{i}^{\prime}\right)\right)^{2}}
$$

where $y_{i}$ is the true value of the $\mathrm{i}$-th sample, $y_{i}^{\prime}$ is the prediction result of the $i$-th sample, and $n$ is the total number of samples.

\subsubsection{Random Forest (RF) Regression}

RF regression was implemented to build prediction models for fresh AGB and dry AGB. The RF regression model combines a large number of decision trees (ntree) that are used for classification or regression [13,15]. Each tree depends on the values of a random vector sampled independently with the same distribution for all trees in the forest [23]. Similar to ML, $80 \%$ of the total data were used for calibration, and the remaining $20 \%$ were used for validation. The important features for AGB were identified following the method found by de Andrade et al. [43] using the 'RReliefF' package in R. The importance of all predictors was represented by the increase in the mean square error (MSE). By randomly selecting a predictor variable, if the variable is randomly replaced, the error of the model will increase [7].

\subsubsection{Multilayer Perceptron (MLP) Neural Network}

The multilayer perceptron (MLP), also called an artificial neural network (ANN), is mainly constructed by the input and output layers with multiple hidden layers in between [36]. It is a feed-forward neural network that has been indicated to be a general function approximation method that can be used to fit complex functions or solve classification problems [44]. MLP is a fully connected multilayered feed-forward network that contains an input layer, one or more hidden layers and one output layer [44,45]. In this study, MLP was implemented to build prediction models for fresh AGB and dry AGB. Comparing the estimation accuracy of the three models of MLP, ML and RF, the method with higher accuracy was used to construct the estimation model of the AGB.

The MLP model introduces the rectified linear unit (ReLU) activation function, also known as the modified linear unit, which has the advantage of more efficient gradient descent and a simplified calculation process [36]. The formula is as follows:

$$
\operatorname{ReLU}(x)= \begin{cases}x & \text { if } x>0 \\ 0 & \text { if } x \leq 0\end{cases}
$$

\subsubsection{Model Performance Evaluation}

The performance of the linear regression, RF and MLP models was evaluated by comparing the coefficients of determination $\left(R^{2}\right)$ and root square error (RMSE) in predictions [7]. The higher the $R^{2}$ is and the lower the RMSE is, the better the accuracy and effect of the models are [13]. The $R^{2}$ and $R M S E$ were calculated using the following equations:

$$
R^{2}=1-\frac{\sum_{i=1}^{n}\left(y_{i}-y_{i}^{\prime}\right)^{2}}{\sum_{i=1}^{n}\left(y_{i}-\bar{y}_{i}\right)^{2}}
$$




$$
\begin{gathered}
\text { RMSE }=\left[\frac{1}{n} \sum_{i=1}^{n}\left(y_{i}-y_{i}^{\prime}\right)^{2}\right]^{\frac{1}{2}} \\
\text { rRMES }=\frac{R M S E}{\bar{y}_{i}} \\
\text { Bias }=\frac{\sum_{i=1}^{n}\left(y_{i}^{\prime}-y_{i}\right)}{n} \\
\text { rBias }=\frac{\text { Bias }}{\bar{y}} \times 100
\end{gathered}
$$

where $y_{i}$ is the true value of the $\mathrm{i}$-th sample, $y_{i}^{\prime}$ is the prediction result of the $i$-th sample, $\bar{y}_{i}$ is the actual measured average value of the sample, and $n$ is the total number of samples. All modeling was performed using the R programming language in RStudio.

\section{Results}

\subsection{U-Net Model Training and Image Segmentation}

The data collected from the visual information collection platform for $T$. sinensis seedlings are shown in Figure 3, including a normal RGB three-channel color image (Figure 3a) and a depth image corresponding to the RGB image (Figure 3c). In the depth image, the depth value of each pixel was the distance from the object in the scene to the camera.
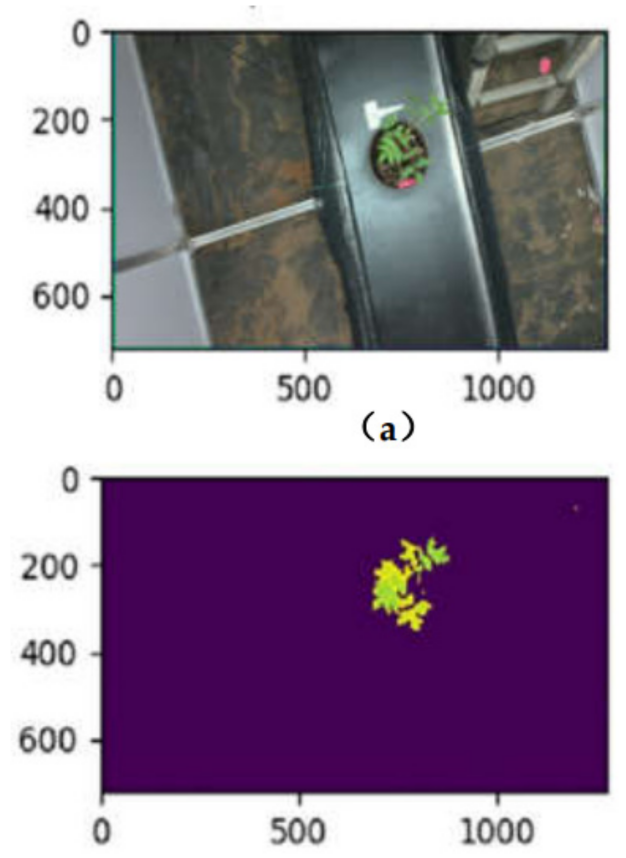

(c)
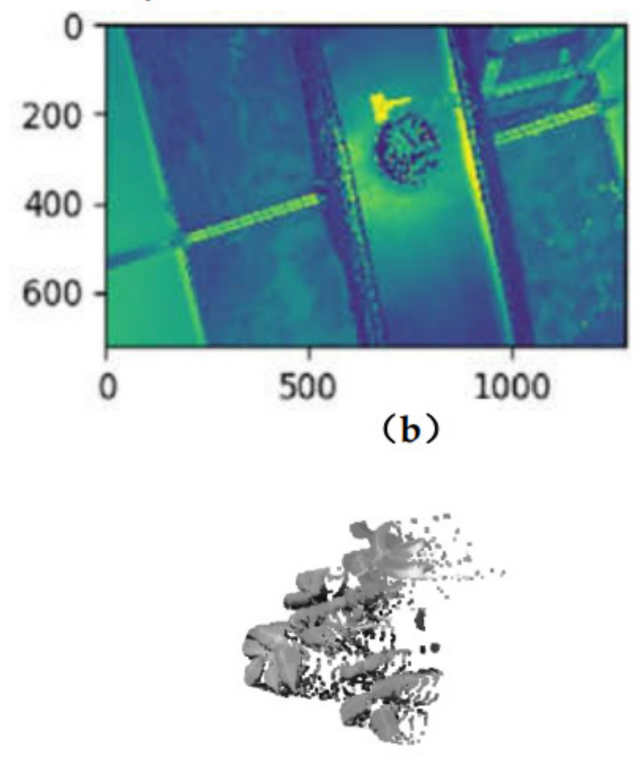

(d)

Figure 3. Original RGB image for a single T. sinensis seedling (a), grayscale image for T. sinensis seedling (b), depth image with depth information (c) and 3D point cloud image (d).

The U-Net network was used to segment the images of T. sinensis seedlings, and the segmentation results are shown in Figure 4. The calibration and validation sets were input into the network together. The calibration set was used for training features, and the validation set was used to find the optimal network model. The test accurately segmented the crown of $T$. sinensis seedlings. Figure $4 \mathrm{~b}-\mathrm{d}$ represents the color values of the pixels of the red, green and blue channels, respectively. Combining the two-dimensional semantic segmentation results and depth information to perform RGB-D three-dimensional recon- 
struction, the 3D point cloud image result was shown in Figure 3d, which was beneficial to the determination of parameters such as plant height and improves the accuracy of the model.

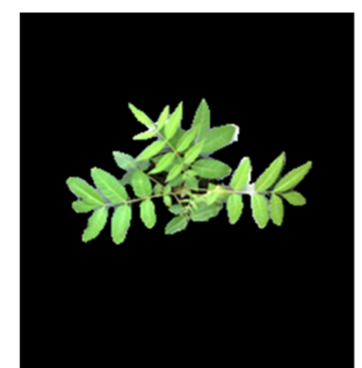

(a)

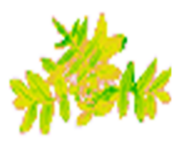

(b)

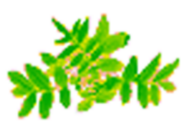

(c)

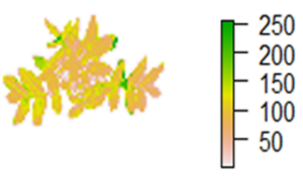

(d)

Figure 4. Image segmentation results of T. sinensis seedlings based on the U-Net model. T. sinensis seedling original image (a), R channel image (b), G channel image (c), B channel image (d).

\subsection{Measured Data of T. sinensis Seedlings}

The statistical analysis results of plant height, fresh AGB and dry AGB are shown in Table 2. The difference between the maximum and minimum plant height was $20.9 \mathrm{~cm}$, the dry AGB range was $0.11-0.96 \mathrm{~g}$, and the fresh AGB range was $0.25-4.51 \mathrm{~g}$. The selection of different $T$. sinensis varieties and the setting of different drought stress gradients make it possible to obtain a wide range of data and ensure the stability of the model derived from the data.

Table 2. Statistics of ground truth data for T. sinensis seedling plant height and dry and fresh aboveground biomass (AGB).

\begin{tabular}{ccccccccc}
\hline \multirow{2}{*}{ Parameters } & \multicolumn{4}{c}{ Calibration } & \multicolumn{4}{c}{ Prediction } \\
\cline { 2 - 9 } & Min & Max & Mean & SD & Min & Max & Mean & SD \\
\hline Plant height $/(\mathrm{cm})$ & 4.10 & 25.00 & 10.80 & 3.19 & 4.11 & 22.30 & 10.88 & 3.27 \\
Dry AGB $/(\mathrm{g})$ & 0.11 & 0.78 & 0.37 & 0.15 & 0.12 & 0.96 & 0.34 & 0.19 \\
Fresh AGB $/(\mathrm{g})$ & 0.39 & 4.51 & 1.57 & 0.68 & 0.25 & 3.52 & 1.61 & 0.77 \\
\hline
\end{tabular}

After the segmentation of T. sinensis image seedlings was obtained, the visible spectrum features of the image were extracted (normalized pixel values of R, G and B channels), and Pearson correlation analysis was applied to select the best feature. Correlation analysis between the color characteristics of the visible light image and the measured dry and fresh AGB of T. sinensis seedlings was carried out. The results are shown in Table 3. The correlation between ExR and the measured biomass of T. sinensis seedlings was low, i.e., only approximately 0.32 . There was a significant negative correlation between NDI and the measured biomass, and the correlation values with dry and fresh AGB were -0.78 and -0.76 , respectively. There was a significant positive correlation between the other parameters and the measured data; for example, the correlation between dry AGB and gray values reached 0.79 , and the correlation between fresh AGB and GMR was as high as 0.81. Therefore, when constructing the estimation model, the two characteristics of ExR and NDI were eliminated, and the remaining six characteristic indices were retained for the construction of the dry and fresh AGB estimation model. 
Table 3. Correlation analysis between image features and dry and fresh AGB.

\begin{tabular}{cccccccccc}
\hline & Gray & ExG & ExR & ExGR & NGRDI & GMR & GRRI & NDI & Depth \\
\hline Dry AGB $/(\mathrm{g})$ & $0.79^{* * *}$ & $0.75^{* * *}$ & $0.37^{* * *}$ & $0.74^{* * *}$ & $0.74^{* * *}$ & $0.77^{* * *}$ & $0.65^{* * *}$ & $-0.76^{* * *}$ & $0.32^{* * *}$ \\
Fresh AGB $/(\mathrm{g})$ & $0.81^{* * *}$ & $0.78^{* * *}$ & $0.32^{* * *}$ & $0.80^{* * *}$ & $0.77^{* * *}$ & $0.81^{* * *}$ & $0.67^{* * *}$ & $-0.81^{* * *}$ & $0.36^{* * *}$ \\
\hline
\end{tabular}

*** Significant correlation at 0.001 level.

\subsection{Estimation Model of T. sinensis Seedling Height}

It can be confirmed that the loss value stabilizes after 200 iterations (Figure 5a). Thus, approximately 200 epochs were selected as the final iteration. The RGB-D-based SLR plant height prediction model yielded a high prediction accuracy, with $R^{2}$ and RMSE values of 0.72 and $1.89 \mathrm{~cm}$, respectively.

(a)

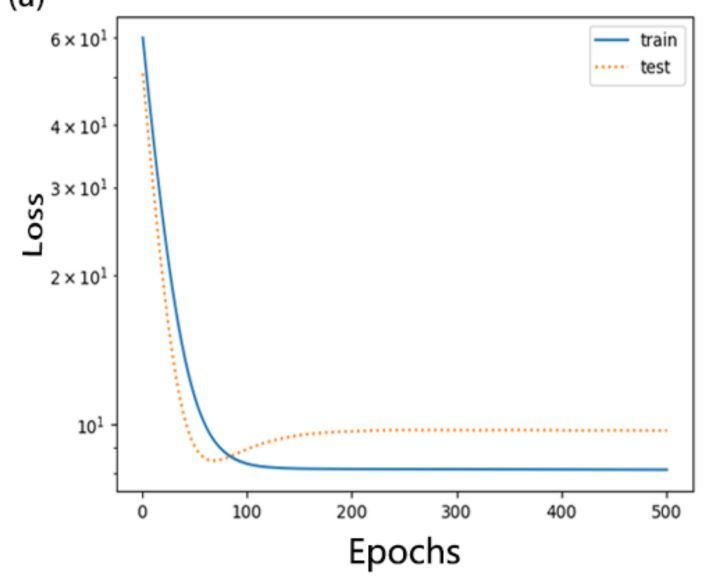

(b)

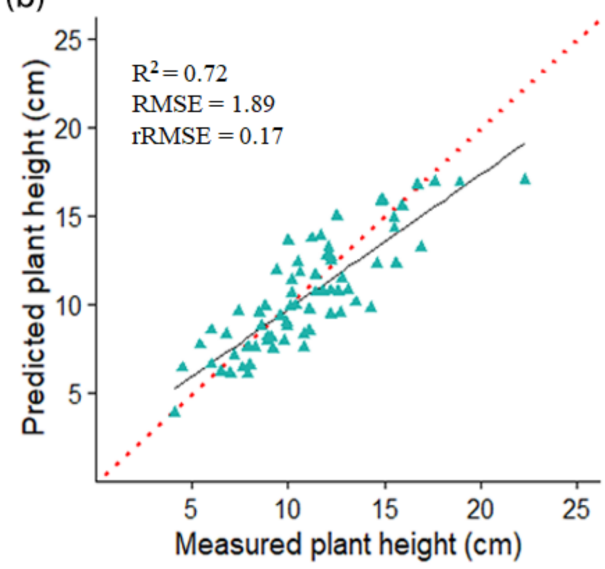

Figure 5. The change curve of the loss function with the number of iterations (a), correlations between the measured truth plant height and the predicted height $(\mathbf{b})$ estimated by the simple linear regression (SLR) model using the RGB-D depth data.

\subsection{Estimation of AGB Using the RF, MLP and ML Models}

\subsubsection{RF Regression Models}

In this study, gray, ExG, ExGR, NGRDI, GMR, GRRI and one depth value derived from RGB-D imagery were used to establish a random forest (RF) regression model for both dry and fresh $T$. sinensis seedlings AGB. The importance of all predictors was evaluated using 'RReliefF' (Figure 6b,d). Evidently, not all predictor variables contributed similarly to dry and fresh AGB. Gray was found to be the most important variable for predicting dry AGB in the RF model (Figure 6b), and GMR was found to be the most important variable for predicting fresh AGB, followed by Gray and GRRI (Figure 6d). Depth contributed less to both dry and fresh AGB. The loss value stabilized when the number of trees reached approximately 500; hence, this value was used as ntree in the RF model. The prediction results for the test dataset showed that the RF models can estimate both dry (Table 4, $R^{2}=0.77$, RMSEP $=0.09 \mathrm{~g}$ ) and fresh (Table $4, R^{2}=0.80$, RMSEP $=0.36 \mathrm{~g}$ ) AGB accurately (Figure 6a,c). 


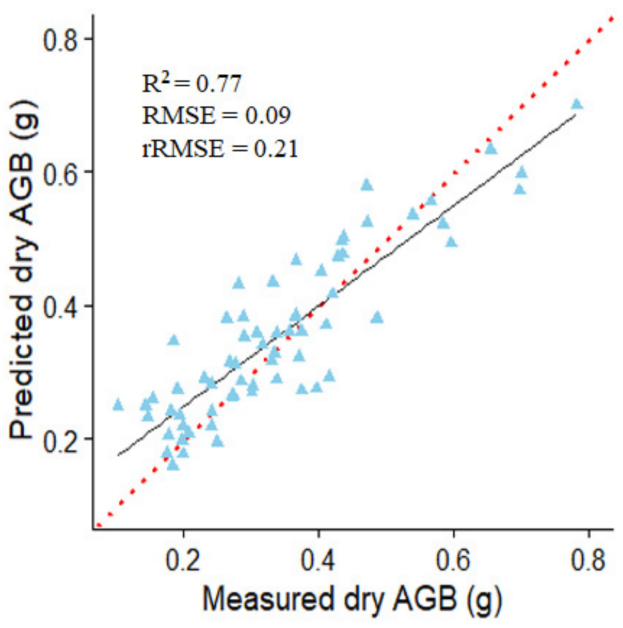

(a)

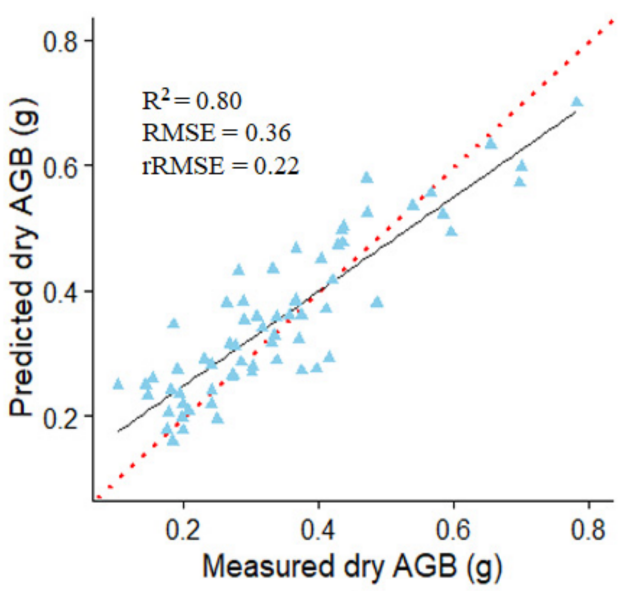

(c)

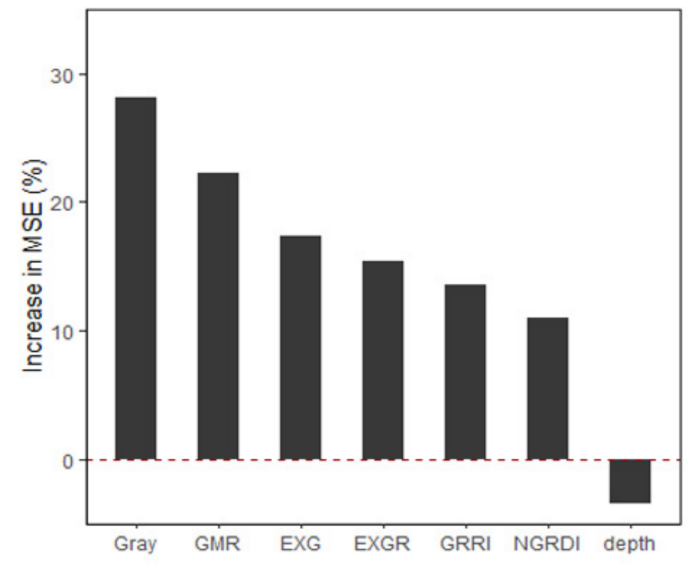

(b)

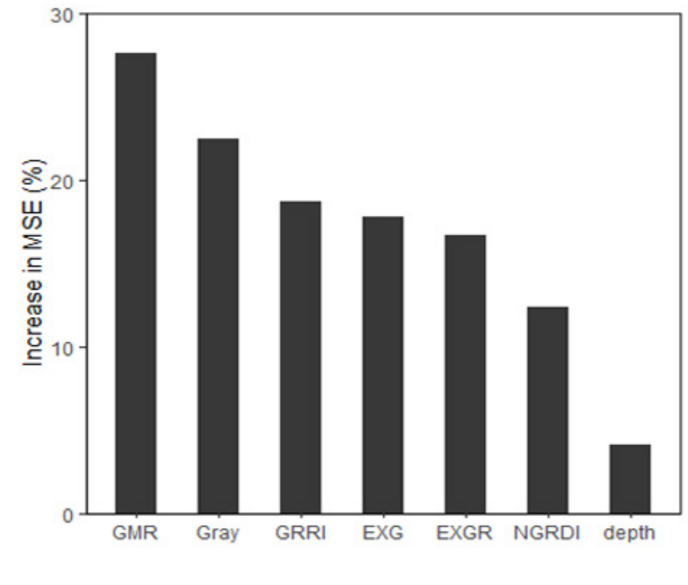

(d)

Figure 6. Prediction of dry AGB using the random forest (RF) regression model (a) and the importance of all predictor variables (b). Prediction of fresh AGB using the RF regression model (c) and the importance of all predictor variables $(\mathbf{d})$.

Table 4. Comparative results for the test dataset of different estimation models.

\begin{tabular}{ccccccc}
\hline Parameter & Models & $\boldsymbol{R}^{\mathbf{2}}$ & RMSE & rRMSE & Bias & rBias/\% \\
\hline Plant Height & Simple linear regression (SLR) & 0.72 & 1.89 & 0.17 & -0.486 & -4.44 \\
& Random Forest (RF) & 0.77 & 0.09 & 0.21 & 0.014 & 4.01 \\
Dry AGB & Multilayer Perceptron (MLP) & 0.77 & 0.07 & 0.20 & 0.001 & 0.36 \\
& Multivariate linear (ML) & 0.76 & 0.10 & 0.28 & -0.020 & -5.63 \\
& Random Forest (RF) & 0.80 & 0.36 & 0.22 & -0.078 & -4.86 \\
Fresh AGB & Multilayer Perceptron (MLP) & 0.83 & 0.32 & 0.20 & -0.008 & -0.55 \\
& Multivariate linear (ML) & 0.78 & 0.33 & 0.22 & -0.049 & -3.27 \\
\hline
\end{tabular}

\subsubsection{MLP and ML Regression Models}

Since the depth value had less effect on the prediction model (Figure $6 b$,d), it was removed when constructing the multilayer perceptron (MLP) and multivariate linear (ML) regression models, and the remaining six sets of visible spectrum indicators were used for modeling. The results show that the three models (RF, MLP, ML) had similar accuracy in the estimation of dry AGB of $T$. sinensis seedlings (Figure 7a,b), with $R^{2}$ values ranging from 0.76 to 0.77 and RMSE values ranging from $0.07 \mathrm{~g}$ to $0.10 \mathrm{~g}$, respectively. MLP produced 
the best accuracy $\left(R^{2}=0.83, R M S E=0.32 \mathrm{~g}\right)$ in the prediction of fresh AGB compared to the other two models.

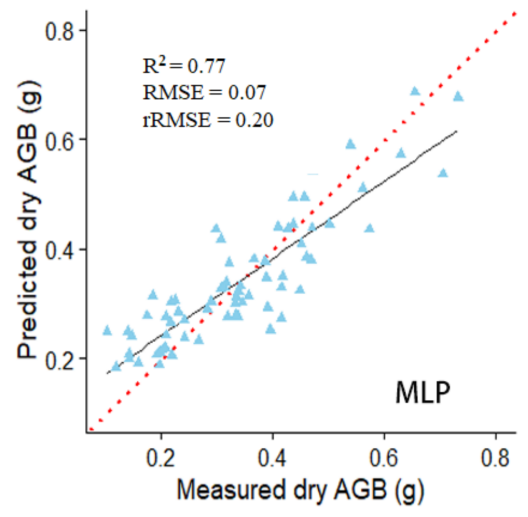

(a)

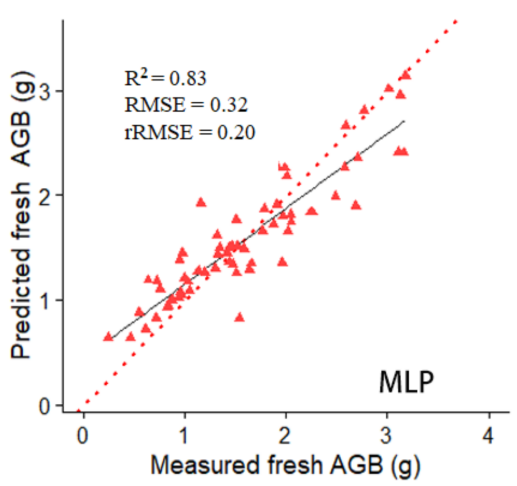

(c)

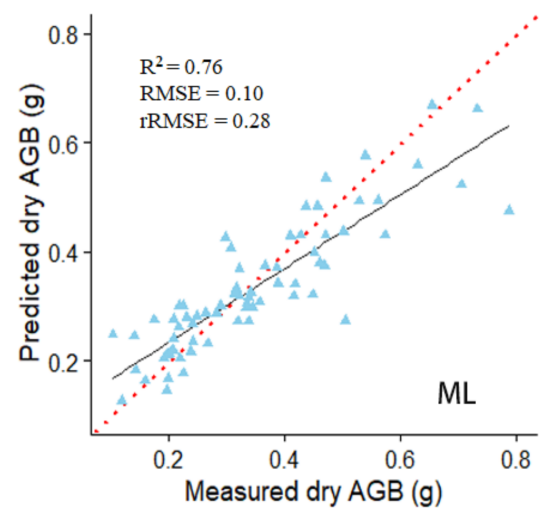

(b)

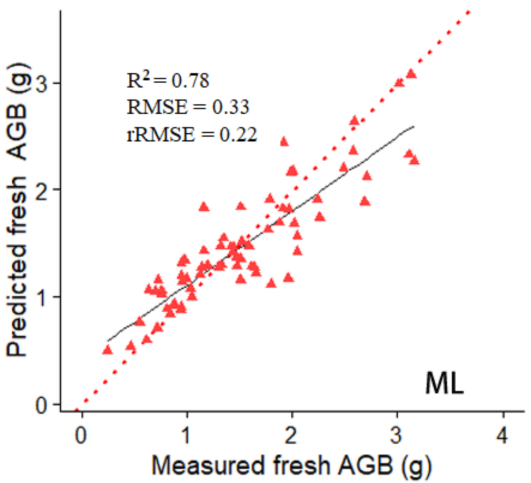

(d)

Figure 7. Measured and predicted dry $(\mathbf{a}, \mathbf{b})$ and fresh $(\mathbf{c}, \mathbf{d})$ AGB using the multilayer perceptron (MLP) and multivariate linear (ML) regression models.

\section{Discussion}

Through the measurement and analysis of plant-related traits, that is, plant phenotype analysis, it is possible to understand the state of plants under stress and the differences in the response of different plants to stress $[17,46]$. In recent years, research on plant phenotypes supported by remote sensing, unmanned aerial vehicles (UAVs) and RGB cameras has received increasing attention $[6,19]$. The application of these technologies can not only reduce the impact of destructive experiments on the environment but also save time and effort and play an important role in accurate and real-time monitoring [46]. Previous studies have been based on field experiments, with less research on the use of RGB-D images to intelligently monitor the growth of potted seedlings. In this study, RGB-D imaging technology was used to obtain images of a single T. sinensis seedling, and the UNet model was used to achieve accurate segmentation of plants and complex backgrounds. Twenty $T$. sinensis varieties were planted under different water stress treatments, and different plant heights and AGB data were provided to ensure the accuracy of model construction. ML, RF, MLP and other models were used to estimate the plant height and AGB of T. sinensis under drought stress. The estimated results showed promising and reliable results for the prediction of plant height and AGB of T. sinensis seedlings.

The essence of plant image segmentation is to classify pixels [45]. Traditional image segmentation technology only uses image grayscale feature information, which can achieve better segmentation effects in single leaves and simple scenes; however, it is not ideal for solving segmentation effects in complex backgrounds [30]. Due to the greater impact of background noise and environmental scenes and poor system robustness, traditional methods cannot provide effective semantic information [47]. With the development of modern high-performance computing, convolutional neural networks have been applied to 
image segmentation [47]. U-Net is a very common semantic segmentation network that is mostly used in medical and pathological image segmentation [44,46]. Zhao et al. [30] used the U-Net method to segment and count the canopy coverage area of Populus euphratica by UAV images, and the segmentation accuracy reached $94.1 \%$. In our experiment, the U-Net network was used to segment the images of T. sinensis seedlings. Similar to previous studies, we also used strategies such as data enhancement and feature visualization to improve the performance of the U-Net model [44]. The results show that the model had strong applicability and can be applied to the segmentation of $T$. sinensis seedlings and complex backgrounds. Plant height is an important phenotypic trait [48]. It can not only be used as a parameter for predicting traits such as biomass but can also reflect the stress state of plants and the difference in response of different plants to stress [49]. In recent years, depth information has gradually been applied to the automatic measurement of plant height, which overcomes the problem of time-consuming and labor-intensive manual measurements that are not suitable for large-scale experiments [50]. Using RGB$\mathrm{D}$ imaging to measure plant height is not new, and current studies have shown that, compared with RGB and grayscale images, depth images contain more accurate geometric feature information [50]. Most of the existing studies are based on nDSM and DEM methods to obtain plant height information [7]. This is different from the research method of Jiang et al. [50], which images with GPS information could not be obtained through 'GPhenoVision' software. Therefore, it was necessary to artificially select the flowerpot mouth as the lowest point of the aboveground part of the plant. In our research, depth images and RGB images were combined to extract Toon seedling images with depth information, and to get its point cloud information, build a 3D point cloud image. The simple linear regression (SLR) analysis result between the depth value and the manual measurement showed a high accuracy with an $\mathrm{R}^{2}$ value of 0.72 , which indicated that this result can provide an accurate height measurement. The measurement Bias and rBias of plant height were $-0.486 \mathrm{~cm}$ and $-4.44 \%$, respectively, indicating that the predicted height was less than the measured height. The reason for this phenomenon was that when obtaining point cloud data, artificially select the flowerpot mouth as the lowest point of the aboveground part of the plant. However, the soil surface layer was used as the lowest point in the actual measurement. In future work, we can use structural metrics from point clouds from LiDAR technology to obtain more accurate point cloud information to determine the accurate lowest point data so as to improve the accuracy of the model [26].

The combination of Gray, GMR and ExG was identified as the key predictor by RReliefF for predicting dry AGB. GMR, Gray and GRRI were the most important in predicting fresh AGB (Figure 6). Research by Freeman et al. [51] found that plant height was highly correlated with AGB and that height improved the accuracy of AGB prediction as an important variable. Inconsistent with previous studies, the depth value was found to be the least important for the prediction of dry and fresh AGB in our study. The reason is that drought stress can cause a series of physiological and biochemical reactions in plants and changes in plant morphology and structure, such as reduced leaf area and limited biomass synthesis but less change in plant height [17]. Plant height and AGB cannot have a significant positive correlation under normal conditions [52]. At this time, plant height cannot be used as an important factor affecting AGB synthesis. Bendig et al. [53] found that cultivar difference is one constraint for biomass prediction by crop height. Similarities to previous results, which can most likely be attributed to the multiple varieties of Toon used in this experiment, lead to increased error [7].

The RF, MLP and ML regression methods were successfully applied to dry and fresh AGB estimation using the visible light spectrum index as the predictor variable $[8,19,44]$. As shown in Table 4, when using MLP to predict the dry and fresh AGB of T. sinensis seedlings, the bias was the smallest, both close to zero. Our results show that the MLP model has the best accuracy in predicting AGB, followed by the RF regression model. Compared with the first two models, the performance of the ML regression model was slightly worse, which is consistent with the results of previous studies [23]. It has been shown that MLP trained with 
a backpropagation algorithm takes advantage of dealing with limited samples, and highdimensional datasets have been capable of discovering relationships in datasets [8,36]. RF has good anti-noise ability but also introduces randomness and is not easy to overfit [8]. The ML regression model fitting result is more intuitive and faster; however, in most cases, the calculation is complicated and cannot solve the problems concerning multicollinearity [36], while MLP and RF methods are more suitable for smaller samples [8]. Consequently, future study is required to increase the sample size of different growth stages and different varieties of $T$. sinensis seedlings to improve the performance and accuracy of the current three regression models. In our study, the machine learning models (RF, MLP and ML) coupled with the visible spectrum (VIS) index had better performance in predicting fresh AGB $\left(R^{2}\right.$ value is $\left.0.78-0.83\right)$ under different drought stress treatments compared with the prediction results of dry AGB. These results indicate that the VIS region is sensitive to fresh matter and vegetation water content [14]. In contrast, many studies have shown that NIRs are the most sensitive area for AGB [36]. Yang, Li, Wang and Yu [14] found that the red edge position $(670-760 \mathrm{~nm})$ had better performance in estimating plant AGB. Therefore, in a follow-up study, it is essential to design experiments and use cameras with more spectral bands to obtain the vegetation index as a variable to understand whether it has an impact on the prediction accuracy of AGB. Based on RedEdge-M and LiDAR data, Zhu et al. [26] used random forest (RF), backpropagation neural network (BP), and support vector machine (SVM) model construction methods to study the performance of the AGB estimation models. The $R^{2}$ values of methods for estimating fresh AGB using RedEdge$\mathrm{M}$ data and LiDAR data were $0.67-0.77$ and $0.70-0.85$, respectively. In this experiment, the prediction results of different models $R^{2}$ were between $0.76-0.83$, the maximum bias was -0.079 , and the minimum was 0.001 . The above results show that the estimation model used in this experiment has certain advantages in predicting AGB. In subsequent experiments, the accuracy of the model can be further verified by adding RedEdge and LiDAR data to continuously improve the accuracy of the model.

\section{Conclusions}

In this study, RGB-D imaging technology was used to obtain RGB color and depth image data of $T$. sinensis seedlings to estimate the plant height and aboveground biomass through three regression methods (ML, RF and MLP). Simple linear regression (SLR) yields a high prediction accuracy $\left(R^{2}=0.72, \operatorname{RMSEP}=1.89 \mathrm{~cm}\right)$ for plant height. The combination of Gray, GMR and ExG was identified as the key predictor by RReliefF for the prediction of dry AGB. GMR was the most important variable in the prediction of fresh AGB. Additionally, all three regression methods performed well in the AGB estimation. MLP produced the best prediction accuracy in predicting dry and fresh aboveground biomass compared to the other two regression models, with yields $R^{2}$ values of 0.77 and 0.83 , respectively.

This suggests that RGB-D imaging could be a promising technique for predicting the height and AGB of T. sinensis seedlings. This technology can be used for the realtime dynamic management of $T$. sinensis seedlings, to obtain high-quality raw materials and medicinal.

Author Contributions: Y.L. and W.L. conceived the ideas and designed the methodology; W.L. collected and analyzed the data and wrote the manuscript; Y.L. guided the data analysis and reviewed the manuscripts; J.L. and J.J. supervised all stages of the experiment. All authors have read and agreed to the published version of the manuscript.

Funding: This research was supported by the Zhejiang Science and Technology Major Program on Agricultural New Variety Breeding (2021C02070-1) and the Fundamental Research Funds of CAF (CAFYBB2020SZ004-3).

Institutional Review Board Statement: Not applicable.

Informed Consent Statement: Not applicable. 


\section{Data Availability Statement: Not applicable.}

Conflicts of Interest: The authors declare no conflict of interest.

\section{References}

1. Peng, W.; Liu, Y.; Hu, M.; Zhang, M.; Yang, J.; Liang, F.; Huang, Q.; Wu, C. Toona sinensis: A comprehensive review on its traditional usages, phytochemisty, pharmacology and toxicology. Rev. Bras. Farmacogn. 2019, 29, 111-124. [CrossRef] [PubMed]

2. Cao, J.-J.; Lv, Q.-Q.; Zhang, B.; Chen, H.-Q. Structural characterization and hepatoprotective activities of polysaccharides from the leaves of Toona sinensis (A. Juss) Roem. Carbohydr. Polym. 2019, 212, 89-101. [CrossRef] [PubMed]

3. Chen, H.-D.; Yang, S.-P.; Wu, Y.; Dong, L.; Yue, J.-M. Terpenoids from Toona ciliata. J. Nat. Prod. 2009, 72, 685-689. [CrossRef]

4. Shi, Q.-Q.; Zhang, X.-J.; Wang, T.-T.; Zhang, Y.; Zeb, M.A.; Zhang, R.-H.; Li, X.-L.; Xiao, W.-L. Toonaones A- I, limonoids with NLRP3 inflammasome inhibitory activity from Toona ciliata M. Roem. Phytochemistry 2021, 184, 112661. [CrossRef] [PubMed]

5. Li, P.; Zhan, X.; Que, Q.; Qu, W.; Liu, M.; Ouyang, K.; Li, J.; Deng, X.; Zhang, J.; Liao, B. Genetic diversity and population structure of Toona ciliata Roem. based on sequence-related amplified polymorphism (SRAP) markers. Forests 2015, 6, 1094-1106. [CrossRef]

6. Maimaitijiang, M.; Ghulam, A.; Sidike, P.; Hartling, S.; Maimaitiyiming, M.; Peterson, K.; Shavers, E.; Fishman, J.; Peterson, J.; Kadam, S. Unmanned Aerial System (UAS)-based phenotyping of soybean using multi-sensor data fusion and extreme learning machine. ISPRS J. Photogramm. Remote Sens. 2017, 134, 43-58. [CrossRef]

7. Li, B.; Xu, X.; Zhang, L.; Han, J.; Bian, C.; Li, G.; Liu, J.; Jin, L. Above-ground biomass estimation and yield prediction in potato by using UAV-based RGB and hyperspectral imaging. ISPRS J. Photogramm. Remote Sens. 2020, 162, 161-172. [CrossRef]

8. Zhou, X.; Zhu, X.; Dong, Z.; Guo, W. Estimation of biomass in wheat using random forest regression algorithm and remote sensing data. Crop J. 2016, 4, 212-219.

9. Avolio, M.L.; Hoffman, A.M.; Smith, M.D. Linking gene regulation, physiology, and plant biomass allocation in Andropogon gerardii in response to drought. Plant Ecol. 2018, 219, 1-15. [CrossRef]

10. Holman, F.H.; Riche, A.B.; Michalski, A.; Castle, M.; Wooster, M.J.; Hawkesford, M.J. High throughput field phenotyping of wheat plant height and growth rate in field plot trials using UAV based remote sensing. Remote Sens. 2016, 8, 1031. [CrossRef]

11. Walter, J.; Edwards, J.; McDonald, G.; Kuchel, H. Photogrammetry for the estimation of wheat biomass and harvest index. Field Crop. Res. 2018, 216, 165-174. [CrossRef]

12. Ballesteros, R.; Ortega, J.F.; Hernandez, D.; Moreno, M.A. Onion biomass monitoring using UAV-based RGB imaging. Precis. Agric. 2018, 19, 840-857. [CrossRef]

13. Capolupo, A.; Kooistra, L.; Berendonk, C.; Boccia, L.; Suomalainen, J. Estimating plant traits of grasslands from UAV-acquired hyperspectral images: a comparison of statistical approaches. ISPRS Int. J. Geo-Inf. 2015, 4, 2792-2820. [CrossRef]

14. Yang, H.; Li, F.; Wang, W.; Yu, K. Estimating Above-Ground Biomass of Potato Using Random Forest and Optimized Hyperspectral Indices. Remote Sens. 2021, 13, 2339. [CrossRef]

15. Laurin, G.V.; Chen, Q.; Lindsell, J.A.; Coomes, D.A.; Del Frate, F.; Guerriero, L.; Pirotti, F.; Valentini, R. Above ground biomass estimation in an African tropical forest with lidar and hyperspectral data. ISPRS J. Photogramm. Remote Sens. 2014, 89, 49-58. [CrossRef]

16. Han, L.; Yang, G.; Dai, H.; Xu, B.; Yang, H.; Feng, H.; Li, Z.; Yang, X. Modeling maize above-ground biomass based on machine learning approaches using UAV remote-sensing data. Plant Methods 2019, 15, 10. [CrossRef]

17. Briglia, N.; Montanaro, G.; Petrozza, A.; Summerer, S.; Cellini, F.; Nuzzo, V. Drought phenotyping in Vitis vinifera using RGB and NIR imaging. Sci. Hortic. 2019, 256, 108555. [CrossRef]

18. Yu, L.; Xiong, J.; Fang, X.; Yang, Z.; Chen, Y.; Lin, X.; Chen, S. A litchi fruit recognition method in a natural environment using RGB-D images. Bioprocess. Eng. 2021, 204, 50-63. [CrossRef]

19. Rueda-Ayala, V.P.; Peña, J.M.; Höglind, M.; Bengochea-Guevara, J.M.; Andújar, D. Comparing UAV-based technologies and RGB-D reconstruction methods for plant height and biomass monitoring on grass ley. Sensors 2019, 19, 535. [CrossRef] [PubMed]

20. Xu, K.; Zhang, J.; Li, H.; Cao, W.; Zhu, Y.; Jiang, X.; Ni, J. Spectrum-and RGB-D-Based Image Fusion for the Prediction of Nitrogen Accumulation in Wheat. Remote Sens. 2020, 12, 4040. [CrossRef]

21. Teng, X.; Zhou, G.; Wu, Y.; Huang, C.; Dong, W.; Xu, S. Three-Dimensional Reconstruction Method of Rapeseed Plants in the Whole Growth Period Using RGB-D Camera. Sensors 2021, 21, 4628. [CrossRef]

22. Barnea, E.; Mairon, R.; Ben-Shahar, O. Colour-agnostic shape-based 3D fruit detection for crop harvesting robots. Biosyst. Eng. 2016, 146, 57-70. [CrossRef]

23. Lee, H.; Wang, J.; Leblon, B. Using linear regression, Random Forests, and Support Vector Machine with unmanned aerial vehicle multispectral images to predict canopy nitrogen weight in corn. Remote Sens. 2020, 12, 2071. [CrossRef]

24. Marabel, M.; Alvarez-Taboada, F. Spectroscopic determination of aboveground biomass in grasslands using spectral transformations, support vector machine and partial least squares regression. Sensors 2013, 13, 10027-10051. [CrossRef] [PubMed]

25. Axelsson, C.; Skidmore, A.K.; Schlerf, M.; Fauzi, A.; Verhoef, W. Hyperspectral analysis of mangrove foliar chemistry using PLSR and support vector regression. Int. J. Remote Sens. 2013, 34, 1724-1743. [CrossRef]

26. Zhu, W.; Sun, Z.; Peng, J.; Huang, Y.; Li, J.; Zhang, J.; Yang, B.; Liao, X. Estimating maize above-ground biomass using 3D point clouds of multi-source unmanned aerial vehicle data at multi-spatial scales. Remote Sens. 2019, 11, 2678. [CrossRef] 
27. Nguyen, H.T.; Lee, B.-W. Assessment of rice leaf growth and nitrogen status by hyperspectral canopy reflectance and partial least square regression. Eur. J. Agron. 2006, 24, 349-356. [CrossRef]

28. Mutanga, O.; Adam, E.; Cho, M.A. High density biomass estimation for wetland vegetation using WorldView-2 imagery and random forest regression algorithm. Int. J. Appl. Earth Obs. Geoinf. 2012, 18, 399-406. [CrossRef]

29. Liu, Z.; Huang, J. Semantic Segmentation Network of Uav Image Based on Improved U-Net; IOP Publishing Ltd.: Bristol, UK, 2019; p. 052050.

30. Zhao, T.; Yang, Y.; Niu, H.; Wang, D.; Chen, Y. Comparing U-Net convolutional network with mask R-CNN in the performances of pomegranate tree canopy segmentation. In Multispectral, Hyperspectral, and Ultraspectral Remote Sensing Technology, Techniques and Applications VII; International Society for Optics and Photonics: Bellingham, WA, USA, 2018; p. 107801J.

31. Zou, K.; Chen, X.; Zhang, F.; Zhou, H.; Zhang, C. A Field Weed Density Evaluation Method Based on UAV Imaging and Modified U-Net. Remote Sens. 2021, 13, 310. [CrossRef]

32. Niu, Y.; Zhang, L.; Zhang, H.; Han, W.; Peng, X. Estimating above-ground biomass of maize using features derived from UAV-based RGB imagery. Remote Sens. 2019, 11, 1261. [CrossRef]

33. Wang, C.; Du, P.; Wu, H.; Li, J.; Zhao, C.; Zhu, H. A cucumber leaf disease severity classification method based on the fusion of DeepLabV3 ${ }^{+}$and U-Net. Comput. Electron. Agric. 2021, 189, 106373. [CrossRef]

34. Guijarro, M.; Pajares, G.; Riomoros, I.; Herrera, P.J.; Burgos-Artizzu, X.P.; Ribeiro, A. Automatic segmentation of relevant textures in agricultural images. Comput. Electron. Agric. 2011, 75, 75-83. [CrossRef]

35. Kataoka, T.; Kaneko, T.; Okamoto, H.; Hata, S. Crop Growth Estimation System Using Machine Vision; IEEE; Piscataway, NJ, USA, pp. b1079-b1083.

36. Suchacz, B.; Wesołowski, M. The recognition of similarities in trace elements content in medicinal plants using MLP and RBF neural networks. Talanta 2006, 69, 37-42. [CrossRef]

37. Kross, A.; McNairn, H.; Lapen, D.; Sunohara, M.; Champagne, C. Assessment of RapidEye vegetation indices for estimation of leaf area index and biomass in corn and soybean crops. Int. J. Appl. Earth Obs. Geoinf. 2015, 34, 235-248. [CrossRef]

38. Cui, R.-X.; Liu, Y.-D.; Fu, J.-D. Estimation of Leaf Nitrogen Accumulation of Winter Wheat Based on Machine Learning and Visible Light Spectroscopy. Spectrosc. Spectr. Anal. 2016, 36, 1837-1842.

39. Du, M.; Noguchi, N. Monitoring of wheat growth status and mapping of wheat yield's within-field spatial variations using color images acquired from UAV-camera system. Remote Sens. 2017, 9, 289. [CrossRef]

40. Müller, K.; Böttcher, U.; Meyer-Schatz, F.; Kage, H. Analysis of vegetation indices derived from hyperspectral reflection measurements for estimating crop canopy parameters of oilseed rape (Brassica napus L.). Biosyst. Eng. 2008, 101, 172-182. [CrossRef]

41. Yan, J.; Huang, J.H.; He, M.; Lu, H.B.; Yang, R.; Kong, B.; Xu, Q.S.; Liang, Y.Z. Prediction of retention indices for frequently reported compounds of plant essential oils using multiple linear regression, partial least squares, and support vector machine. $J$. Sep. Sci. 2013, 36, 2464-2471. [CrossRef] [PubMed]

42. Bottou, L. Large-scale machine learning with stochastic gradient descent. In Proceedings of the COMPSTAT'2010, Paris, France, 22-27 August 2010; pp. 177-186.

43. De Andrade, F.S.; Haga, I.A.; Lyra, M.L.; De Carvalho, T.R.; Haddad, C.F.B.; Giaretta, A.A.; Toledo, L.F. Reassessment of the taxonomic status of Pseudopaludicola parnaiba (Anura, Leptodactylidae, Leiuperinae), with the description of a new cryptic species from the Brazilian Cerrado. Eur. J. Taxon. 2020, 679, 1-36. [CrossRef]

44. Zhang, C.; Pan, X.; Li, H.; Gardiner, A.; Sargent, I.; Hare, J.; Atkinson, P.M. A hybrid MLP-CNN classifier for very fine resolution remotely sensed image classification. ISPRS J. Photogramm. Remote Sens. 2018, 140, 133-144. [CrossRef]

45. Mithun, B.S.; Shinde, S.; Bhavsar, K.; Chowdhury, A.; Mukhopadhyay, S.; Gupta, K.; Bhowmick, B.; Kimbahune, S. NonDestructive Method to Detect Artificially Ripened Banana Using Hyperspectral Sensing and RGB Imaging. In Sensing for Agriculture and Food Quality and Safety X; Kim, M.S., Chao, K., Chin, B.A., Cho, B.K., Eds.; International Society for Optics and Photonics: Bellingham, WA, USA, 2018; Volume 10665.

46. Asaari, M.S.M.; Mishra, P.; Mertens, S.; Dhondt, S.; Inzé, D.; Wuyts, N.; Scheunders, P. Close-range hyperspectral image analysis for the early detection of stress responses in individual plants in a high-throughput phenotyping platform. ISPRS J. Photogramm. Remote Sens. 2018, 138, 121-138. [CrossRef]

47. Smith, A.G.; Petersen, J.; Selvan, R.; Rasmussen, C.R. Segmentation of roots in soil with U-Net. Plant Methods 2020, 16, 13. [CrossRef]

48. Andujar, D.; Ribeiro, A.; Fernández-Quintanilla, C.; Dorado, J. Using depth cameras to extract structural parameters to assess the growth state and yield of cauliflower crops. Comput. Electron. Agric. 2016, 122, 67-73. [CrossRef]

49. Jiang, Y.; Li, C.; Paterson, A.H.; Sun, S.; Xu, R.; Robertson, J. Quantitative analysis of cotton canopy size in field conditions using a consumer-grade RGB-D camera. Front. Plant Sci. 2018, 8, 2233. [CrossRef]

50. Jiang, Y.; Li, C.; Paterson, A.H. High throughput phenotyping of cotton plant height using depth images under field conditions. Comput. Electron. Agric. 2016, 130, 57-68. [CrossRef]

51. Freeman, K.W.; Girma, K.; Arnall, D.B.; Mullen, R.W.; Martin, K.L.; Teal, R.K.; Raun, W.R. By-plant prediction of corn forage biomass and nitrogen uptake at various growth stages using remote sensing and plant height. Agron. J. 2007, 99, 530-536. [CrossRef] 
52. Guo, J.; Yang, Y.; Wang, G.; Yang, L.; Sun, X. Ecophysiological responses of Abies fabri seedlings to drought stress and nitrogen supply. Physiol. Plant. 2010, 139, 335-347.

53. Bendig, J.; Bolten, A.; Bennertz, S.; Broscheit, J.; Eichfuss, S.; Bareth, G. Estimating biomass of barley using crop surface models (CSMs) derived from UAV-based RGB imaging. Remote Sens. 2014, 6, 10395-10412. [CrossRef] 